\title{
Quantitative Inference of Cellular Parameters From Microfluidic Cell Culture Systems
}

\author{
Khamir Mehta, ${ }_{1}^{1}$ Geeta Mehta, ${ }^{2}$ Shuichi Takayama, ${ }^{2}$ Jennifer Linderman ${ }^{1,2}$ \\ ${ }^{1}$ Department of Chemical Engineering, University of Michigan, 3328 G.G. Brown, \\ 2300 Hayward, Ann Arbor, Michigan 48109; telephone: 734-763-0679; \\ fax: 734-764-7453; e-mail: linderma@umich.edu \\ ${ }^{2}$ Department of Biomedical Engineering, University of Michigan, Ann Arbor, Michigan \\ Received 5 December 2008; revision received 9 March 2009; accepted 17 March 2009 \\ Published online 27 March 2009 in Wiley InterScience (www.interscience.wiley.com). DOI 10.1002/bit.22334
}

ABSTRACT: Microfluidic cell culture systems offer a convenient way to measure cell biophysical parameters in conditions close to the physiological environment. We demonstrate the application of a mathematical model describing the spatial distribution of nutrient and growth factor concentrations in inferring cellular oxygen uptake rates from experimental measurements. We use experimental measurements of oxygen concentrations in a poly(dimethylsiloxane) (PDMS) microreactor culturing human hepatocellular liver carcinoma cells (HepG2) to infer quantitative information on cellular oxygen uptake rates. We use a novel microchannel design to avoid the parameter correlation problem associated with simultaneous cellular uptake and diffusion of oxygen through the PDMS surface. We find that the cellular uptake of oxygen is dependent on the cell density and can be modeled using a logistic term in the Michaelis-Menten equation. Our results are significant not only for the development of novel assays to quantitatively infer cell response to stimuli, but also for the development, design, and optimization of novel in vitro systems for drug discovery and tissue engineering.

Biotechnol. Bioeng. 2009;103: 966-974.

(C) 2009 Wiley Periodicals, Inc.

KEYWORDS: oxygen uptake; microfluidic reactor; parameter inference; PDMS; mass transfer coefficient; mathematical model

\section{Introduction}

Microfluidics-based cell culture systems offer an inexpensive and attractive option for culturing cells in conditions closer to physiological than static cultures (e.g., small ratio of fluid volume to cell volume, fluid flow) and hence are favored for various applications in biomedicine (Andersson and van

Correspondence to: J. Linderman

Contract grant sponsor: US Army Research Laboratories and Research Office

Contract grant number: DAAD 19-03-1-0168 den Berg, 2004; Fisher and Peattie, 2007; Mehta et al., 2008; Puleo et al., 2007). Recent advances in microchannel cell culture bioreactors have increased our ability to accurately characterize the microenvironment in such systems by enabling control over the perfusion rate and measurement of molecular concentrations in small samples ( $\mathrm{Gu}$ et al., 2004; Situma et al., 2006). These advances have resulted in application of microfluidics-based systems to, for example, liver tissue engineering and drug discovery (Kang et al., 2008; Nahmias et al., 2007; Viravaidya et al., 2004). However, the use of such systems for quantitative characterization of cellular responses remains limited. An inference procedure that can combine the mathematical description (model) of processes occurring in microchannels with relevant experimental measurements and subsequently extract quantitative information on key cellular parameters would be of great significance in deciphering signaling pathways, optimizing microfluidic devices for tissue engineering applications, and designing assays for pharmacological applications. In this work, we focus on obtaining cellular parameters describing oxygen uptake.

Oxygen is a metabolic and signaling molecule in cell culture systems, and the oxygen consumption rate is an important metric to ascertain culture viability (Hynes et al., 2006). Information on the oxygen uptake rate of a cell culture is useful for designing microchannel cell culture systems and also in development of various biomedical devices including Bio-artificial liver (Balis et al., 1999; Ostrovidov et al., 2004; Park et al., 2005). Typically, oxygen consumption rates in static cell cultures are measured by tracking the oxygen concentration in the culture media for a regulated external oxygen partial pressure (Foy et al., 1994; Guarino et al., 2004; Rotem et al., 1992). The applicability of the uptake rates measured in standard static cultures to physiological conditions remains unknown. Recently, experimental techniques have been developed to measure oxygen concentrations within microfluidic devices (Mehta et al., 2007; Sin et al., 2004; Sud et al., 2006). Mathematical 
models have also been constructed to analyze the oxygen transfer and requirements for such perfusion based cell culture systems (Ghanem and Shuler 2000; Mehta and Linderman 2006; Roy et al., 2001). However, there has been little effort in using these models in their inverse form and developing quantitative inference strategies to allow determination of cellular oxygen uptake parameters from experimental data. In this work, we demonstrate modelbased inference of cellular uptake parameters; in the future the pairing of quantitative assays with inference may allow the study of other cellular responses.

Microfluidics-based cell culture systems are typically made from poly(dimethylsiloxane) (PDMS) in part due to its high oxygen permeability. However, the inference of oxygen uptake rates from the experimental measurements in PDMS devices is made difficult due to the intrinsic correlation of the model parameters characterizing the diffusion of oxygen from PDMS surface and uptake of oxygen by cells. Here we have developed a simple experimental and computational procedure to determine the oxygen uptake rate of a cellular culture in PDMS microchannels. We demonstrate the performance of our method using measurements of oxygen uptake rate in a PDMS microchannel culturing HepG2 liver cells.

\section{Methods}

\section{Experimental Methods}

\section{Microdevice Fabrication and Assembly}

The microbioreactor was comprised of two compartments, a channel layer and a PDMS-parylene C-PDMS membrane. The device was fabricated as previously described (Mehta et al., 2007). The microdevice design used for the experiments is shown in Figure 1 and has a slanted channel connecting two parallel channels at the center of the device. The cells are seeded in the slanted channel region, which is $200 \mu \mathrm{m}$ high and $300 \mu \mathrm{m}$ wide, while the remaining microchannels measure $30 \mu \mathrm{m}$ high and $300 \mu \mathrm{m}$ wide.

\section{Fluid Flow by Braille Actuation}

An array of 48 pin actuators adapted from a Braille display module (SC9, KGS, Saitama, Japan) was used for fluid actuation (Futai et al., 2006). The pin actuator module was controlled with a computer via Universal Serial Bus (USB) through a finger-sized stand alone custom controller circuit board (Olimex, Plovdiv, Bulgaria; Futai et al., 2006). The microfluidic bioreactor chip interfaces with the pin actuator module by simply holding the chip in place such that the channels align with pins which push upward closing the channel. The pin movements for valving and pumping were controlled with a custom computer program written in C sharp. The average flow rates used for these experiments were in the range of $0.09-31.5 \mu \mathrm{m} / \mathrm{s}$. The flow rates were
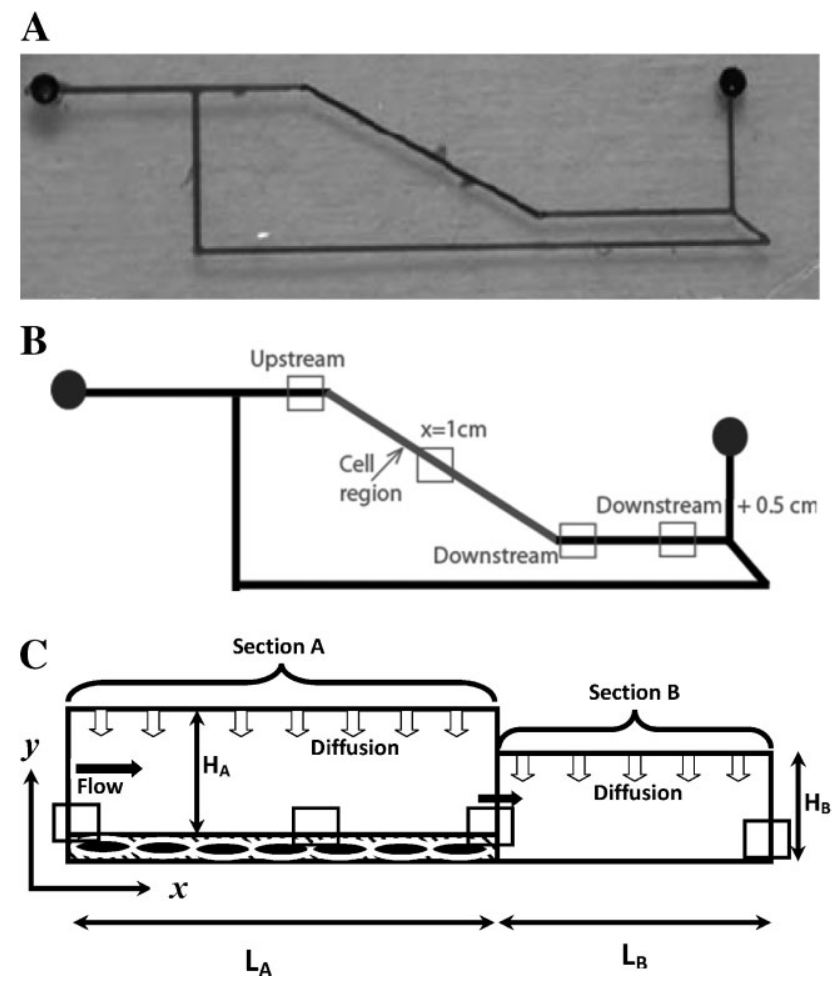

Figure 1. Microfabricated PDMS microreactor. A: Photograph of the device, with food dye in the channels. B: Device schematic with rectangles indicating regions for oxygen measurement. Filled circles indicate the location of the Braille valve pumps. Cells are present only in the portion of the channel highlighted. This channel is $200 \mu \mathrm{m}$ high and $300 \mu \mathrm{m}$ wide; other regions of channel without cells are $30 \mu \mathrm{m}$ high and $300 \mu \mathrm{m}$ wide. C: Model geometry for the PDMS device (side view). The device is divided into two sections, Section A where the cells are cultured and (downstream) Section $B$ where there are no cells. The empty rectangles indicate the oxygen measurement points. The length $L_{A}$ is $17.5 \mathrm{~mm}, L_{B}$ is $5 \mathrm{~mm}$ and the height $H_{A}$ is $200 \mu \mathrm{m}$ and $\mathrm{H}_{\mathrm{B}}$ is $30 \mu \mathrm{m}$.

measured by tracking $6 \mu \mathrm{m}$ diameter fluorescent beads (Carnine, polystyrene microspheres, Molecular Probes, Eugene, OR) using a digital CCD camera (Orca-ER, Hamamatsu Photonics, Hamamatsu, Japan) and a fluorescence stereomicroscope (Nikon SMZ1500), as described in Mehta et al. (2007). The image sequences were acquired at $\sim 18$ frames/s to determine the velocity of the microspheres at the center of the microchannels, which are representative of the fluid velocity, and were used to determine the average fluid flow rate. An entire pumping cycle was used to measure each flow rate in order to compensate for backflow during certain steps of a pumping cycle.

\section{Cell Culture}

HepG2 cells (human hepatocellular carcinoma, ATCC, HB8065) were cultured in Dulbecco's Modified Eagle's Medium (DMEM, 11960, Gibco, Grand Island, NY) with 15\% Fetal bovine serum (FBS, 10082, Gibco), 1\% (v/v) antibiotic-antimicotic (15240, Gibco) and $1 \% \quad(\mathrm{v} / \mathrm{v})$ 
GlutaMAX2-I Supplement (35050, Gibco) in a humidified $5 \% \mathrm{CO}_{2}$ incubator.

\section{Cell Seeding in Microdevices}

Fibronectin (100 mg/mL, F2006, Sigma, St. Louis, MO) was pipetted into the microdevices to increase cell attachment and followed by a $30 \mathrm{~min}$ absorption period. Media was then introduced to the device and the chip was placed on an array of pin actuators adapted from Braille displays for at least $1 \mathrm{~h}$ to peristaltically pump fluid through the channels. Cells (in DMEM) were seeded onto the chip through the cell seeding ports and directed into the desired location by using Braille pumping and valving as previously described (Mehta et al., 2007). Cells were given $2-4 \mathrm{~h}$ to attach under a no flow condition and then the chip was perfused with media for $12-$ $14 \mathrm{~h}$. The device was maintained at $37^{\circ} \mathrm{C}$ and $5 \% \mathrm{CO}_{2}$.

\section{Oxygen Measurement}

Dissolved oxygen concentration in microdevices was measured in real time using an optics-based lifetime detection technique (Mehta et al., 2007). An oxygen sensitive dye, ruthenium tris $\left(2,2^{\prime}\right.$-dipyridyl $)$ dichloride hexahydrate (RTDP) dissolved in the media was excited by a blue LED in frequency domain by square waves generated by a function generator. The emission signal was captured by a silicon PIN photodiode with preamplifier and the data was acquired on a LabVIEW graphic user interface.

Oxygen concentration was determined at four points in the reactor as indicated in Figure $1 \mathrm{~B}$. We refer to the region where cells are cultured as Section A of the channel (three oxygen measurement points) and the region downstream of the cell as Section B (one oxygen measurement point). Oxygen measurements made in Section B are used to estimate the mass transfer coefficient for oxygen diffusion through PDMS.

\section{Mathematical Model Formulation}

Figure $1 \mathrm{C}$ shows the simplified rectangular geometry used for the model. The steady state concentration of oxygen (c) in the media in the microchannel is described by the reaction-diffusion equation

$$
u_{x} \frac{\partial c}{\partial x}=D_{\mathrm{e}}\left(\frac{\partial^{2} c}{\partial x^{2}}+\frac{\partial^{2} c}{\partial y^{2}}\right)
$$

where $D_{\mathrm{e}}$ is the effective diffusivity of oxygen in the media and $u_{x}$ is the velocity. We assume a low Reynolds number laminar unidirectional flow approximation, and the velocity profile is estimated as (Bird et al., 2001)

$$
u_{x}(y)=6\langle u\rangle \frac{y}{H}\left(1-\frac{y}{H}\right)
$$

where $\langle u\rangle$ is the average velocity and $H$ is the microchannel height. We restrict the problem to two dimensions by neglecting the variation of oxygen concentration along the width. We include the diffusive term in the direction of the flow (axial diffusion) because we consider low media flow rates, although it can be neglected for relatively large flow rates $\left(\langle u\rangle \gg D_{\mathrm{e}} / L\right)$. The inclusion of axial diffusion in the model improves the fit to experimental data and is a significant addition to our previous model (Mehta and Linderman, 2006).

The boundary conditions for Equation (1) in Section A of the device (cell region) are

$$
\begin{gathered}
c(0, y)=c_{\text {in }} \\
\frac{\partial c}{\partial x}(L, y)=0 \\
-D_{\mathrm{e}} \frac{\partial c}{\partial y}(x, 0)=F \\
-D_{\mathrm{e}} \frac{\partial c}{\partial y}(x, H)=k_{\mathrm{la}}\left(c^{*}-c\right)
\end{gathered}
$$

where $c_{\text {in }}$ is the inlet oxygen concentration and $c^{*}$ is the saturation oxygen concentration at the solid (PDMS)liquid (media) interface (assumed to be equal to $8.2 \mathrm{mg} / \mathrm{L}$ ). We use a simple film model for the diffusion process and the supply of oxygen from the top PDMS surface is modeled based on an overall mass transfer coefficient denoted by $k_{\mathrm{la}}$. We assume that there is negligible diffusion from the bottom surface due to the presence of impermeable layer of parylene C. $F$ is the flux of oxygen $\left(\mathrm{mol} / \mathrm{m}^{2} \mathrm{~s}\right)$ corresponding to the cellular uptake and is be given by

$$
F=\mathrm{OUR}_{\mathrm{S}} \phi
$$

where $\mathrm{OUR}_{\mathrm{S}}$ is the specific oxygen uptake rate (uptake rate per cell) and $\phi$ represents the cell density (cells $/ \mathrm{m}^{2}$ ).

In Section B there are no cells at the bottom of the microchannel, and hence that boundary condition (Eq. 5) is modified to the no flux condition

$$
-D_{\mathrm{e}} \frac{\partial c}{\partial y}(x, 0)=0
$$

All other boundary and initial conditions (Eqs 3, 4, and 6) remain as in Section A, with the corresponding inlet concentration for Section B equal to the concentration at the outlet of the Section A. The partial differential equation with nonlinear boundary conditions was solved using FEMLAB ${ }^{\circledR}$ (v3.3 \& v3.4, COMSOL, Inc., Burlington, MA), finite element-based software.

Two possible relationships for the specific oxygen uptake rate $\left(\mathrm{OUR}_{\mathrm{S}}\right)$ are considered here. First, we consider the usual assumption that the specific uptake rate of oxygen follows 
Michaelis-Menten kinetics with parameters $V_{\max }$ and $K_{\mathrm{m}}$ and is independent of cell density (Jorjani and Ozturk 1999; Mehta and Linderman 2006). The total uptake flux of oxygen for the cell culture is assumed to be a sum of the individual uptake rates, for example, a linear function of the total cell density $\phi$ (number of cells per unit area of the channel bottom). This simplest relationship we consider is

$$
\mathrm{OUR}_{\mathrm{S}}=\left(\frac{V_{\max } c}{K_{\mathrm{m}}+c}\right)
$$

We also consider the following alternative relationship. Because the uptake of oxygen by cells is directly related to their growth and cell growth in spatially limited conditions can be affected by the total cell density, the specific uptake rate of oxygen may also depend on cell culture density. To describe this case, we incorporate a logistic term to model the changes in the overall oxygen consumption based on the changes in the growth rate via the total cell density. We hence propose a modified, cell density-dependent specific uptake rate of oxygen as given by

$$
\mathrm{OUR}_{\mathrm{S}}=\left(\frac{V_{\mathrm{max}} c}{K_{\mathrm{m}}+c}\right)\left(1-\frac{\phi}{\phi_{\max }}\right)
$$

The parameter $\phi_{\max }$ represents the maximal cell density that can be cultured in the reactor assuming no nutrient limitations. The inclusion of the logistic growth term was motivated in part by the observed dependence of oxygen consumption on cell density (Cho et al., 2007; Rotem et al., 1992). We use the Bayesian information criteria (BIC) to justify the additional parameter in the model

$$
\mathrm{BIC}=n \ln \left(\frac{\mathrm{RSS}}{n}\right)+k \ln (n)
$$

where $n$ represents the number of observations and RSS is the residual sum of squares (Burnham and Anderson, 2002).

\section{Inferring Parameter Values From Experimental Data}

As described above, we measured oxygen concentrations in the media at four locations inside the bioreactor. The cellular uptake parameters and the mass transfer coefficient ( $V_{\text {max }}, K_{\mathrm{m}}, \phi_{\text {max }}$, and $k_{\mathrm{la}}$ ) are parameters to be learned from this data. The parameter estimation is formulated in terms of a nonlinear least square problem with the objective function $f$ defined as

$$
f=\sum_{i} w_{i}\left(c_{i, \text { pred }}-c_{i, \exp }\right)^{2}
$$

where $c_{i \text {,exp }}$ and $c_{i \text {,pred }}$ are the measured values of concentration of oxygen and the model predicted values of corresponding concentrations for the $i$ th measurement, respectively. The weight parameter $w_{i}$ for each data point can be used to incorporate the relative confidence on the measurement $i$. Parameters values are learned based on minimization of the objective function using a nonlinear least square optimization technique using the LevenbergMarquardt technique in a $\operatorname{MATLAB}^{\mathbb{R}}$ (v. 7.4, 2007, Mathworks, Inc., Natick, MA) platform.

In principle the measurements of oxygen concentrations in Section A are sufficient to learn the parameters ( $V_{\max }, K_{\mathrm{m}}$, $\phi_{\text {max }}$, and $k_{\mathrm{la}}$ ) characterizing the system. However, these parameters are usually highly correlated and it would require modification of the inference procedure and a large number of measurements to learn these parameters with a significant degree of confidence. It is hence advisable to have an independent estimate of at least one of the two parameters $V_{\max }$ and $k_{\text {la }}$, as they have highest degree of correlation. Thus we designed our reactor and experiments to allow measurement of oxygen concentrations in a region containing no cells (Section B). We use the concentration measurements in Section B of the reactor to determine $k_{\text {la }}$ and then use this value and the concentration measurements in Section A to learn $V_{\max }, K_{\mathrm{m}}$, and $\phi_{\max }$.

The goodness of the fit was computed using the standard regression coefficient method calculated as

$$
R^{2}=1-\frac{\sum_{i}\left(c_{i, \mathrm{pred}}-c_{i, \exp }\right)^{2}}{\sum_{i}\left(\bar{c}-c_{i, \exp }\right)^{2}}
$$

where $\bar{c}$ is the mean experimental concentration, and the summation in Equation (12) is on the experimental dataset under consideration. The correlation matrix (Cor) is used to identify parameter values and its elements are computed from the covariance matrix (Cov) given by

$$
\operatorname{Cov}=\sigma_{\mathrm{e}}^{2}\left(J^{\mathrm{T}} J\right)^{-1}
$$

$$
\operatorname{Cor}_{i, j}=\frac{\operatorname{Cov}_{i, j}}{\left(\operatorname{Cov}_{i, i} \operatorname{Cov}_{j, j}\right)^{1 / 2}}
$$

where $n$ is the total number of data points (Bates and Watts 1988; Sadegh Zadeh et al., 2006). $\sigma_{\mathrm{e}}$ is the standard deviation in the residual normalized by the total degrees of freedom of the regression calculated as $n$ minus the number of parameters regressed $(n-p)$ (Donaldson and Schnabel 1987).

\section{Inferring Confidence Intervals for Parameter Values}

The standard errors for this regression problem can be computed using the covariance of parameters in the Cov matrix (Eq. 13); however, considering that we have errors in measurement in both dependent (c) and independent variables (chiefly $\langle u\rangle$ and $\phi$ ), such a calculation can be inaccurate. Because the functional dependence is not explicit, a Monte Carlo approach should be used to compute the standard errors in regression (Alper and Gelb, 
1991; Donaldson and Schnabel, 1987). The procedure for the simulations is followed as per Alper and Gelb (1991). Briefly, new datasets are constructed by sampling the independent variables from their individual distributions constructed from their measured variance, and each of these datasets is used for regression to infer the parameters. The inferred set of parameters can be used to estimate their distributions and also their confidence intervals. Here, we sample the velocity values from a normal distribution with means and the standard deviations estimated from our data. The value of the mass transfer coefficient $\left(k_{\mathrm{la}}\right)$ is estimated for each case using the measured oxygen concentrations in Section B, and subsequently the remaining parameters $\left(V_{\max }, K_{\mathrm{m}}\right.$, and/or $\left.\phi_{\max }\right)$ are inferred for each case of velocity and the $k_{\text {la }}$ value. The confidence interval of the inferred parameters is reported as the standard deviations of the estimates.

\section{Results and Discussion}

\section{Experimental Measurements}

Our experimental data on oxygen concentrations in the microdevice culturing HepG2 cells are shown in Figure 2. There is a drop in the oxygen concentration as we move downstream, corresponding to the uptake of oxygen by cells, and this drop increases with increasing cell density. Increasing the media flow rate $(\langle u\rangle)$ increases the overall concentration of oxygen inside the channel as expected.

The increase in oxygen concentration in Section B of the device (measurement at $x=22.5 \mathrm{~mm}$ ), a region in which cells are absent, provides direct evidence of the diffusive flux of oxygen due to the permeability of the PDMS. Interestingly, the diffusion of oxygen from the ambient air through the highly permeable PDMS devices, although significant, is not sufficient to avoid gradients inside the microchannel.

\section{Quantification of Diffusion}

Cells inside the microchannel are supplied oxygen by two modes-continuous circulation of fresh media and diffusion from the ambient air via the PDMS surface. Quantification of the specific oxygen uptake parameters ( $V_{\max }, K_{\mathrm{m}}$, and possibly $\phi_{\max }$ ) by the cells will entail the characterization of both these modes. The convective supply of oxygen by the media can be calculated from the measured media flow rate and the oxygen solubility, while the diffusion of oxygen from air is calculated based on the estimation of the overall mass transfer coefficient $k_{\mathrm{la}}$. If one attempts to simultaneously infer values of $V_{\max }, K_{\mathrm{m}}$, and $k_{\mathrm{la}}$, the inferred values can be inaccurate due to a high degree of correlation in the parameters. For example, the correlation index $\left(\mathrm{Cor}_{i, j}\right.$ in Eq. 14) between $V_{\max }$ and $k_{\mathrm{la}}$

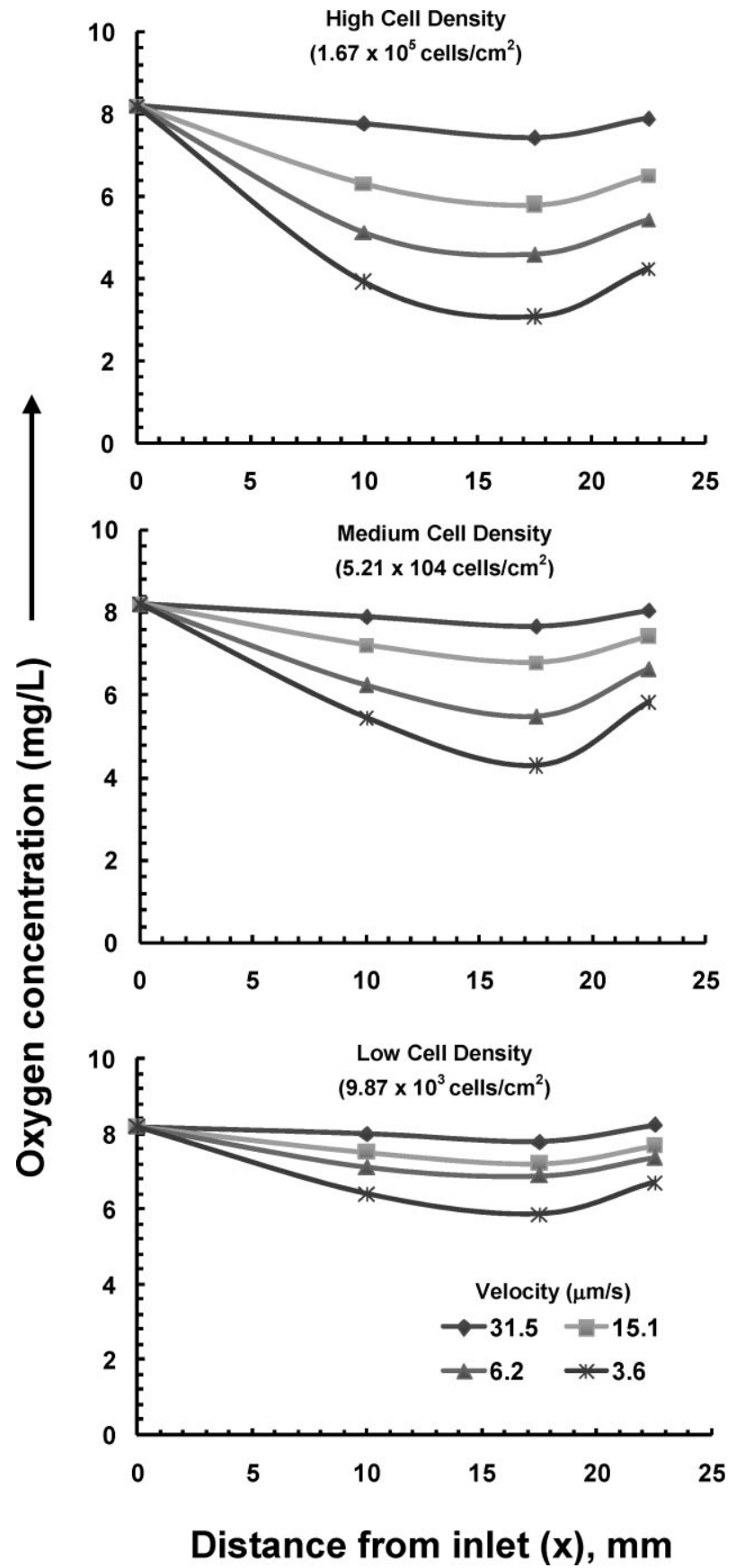

Figure 2. Oxygen concentrations in the microdevice during culture of HepG2 cells. The plots show the experimentally measured oxygen concentration in $\mathrm{mg} / \mathrm{L}$ as a function of distance from the inlet. Experiments were performed at three cell densities: high $\left(1.67 \times 10^{5} \mathrm{cells} / \mathrm{cm}^{2}\right)$, medium $\left(5.2 \times 10^{4} \mathrm{cells} / \mathrm{cm}^{2}\right)$, and low $\left(9.9 \times 10^{3} \mathrm{cells} / \mathrm{cm}^{2}\right)$. Four flow rates were tested and the average fluid velocity $\langle u\rangle$ is indicated. The origin or inlet is defined as the point at which the media first contacts cells as seen in Figure 1.

for the dataset of Figure 2 is $\sim 88 \%$. Hence, we need an independent estimate of at least one of the parameters.

The measurement of the oxygen concentration in Section $\mathrm{B}$ (Fig. 1C) can give an independent estimate of the diffusive flux in the device as in that section there is no uptake of 


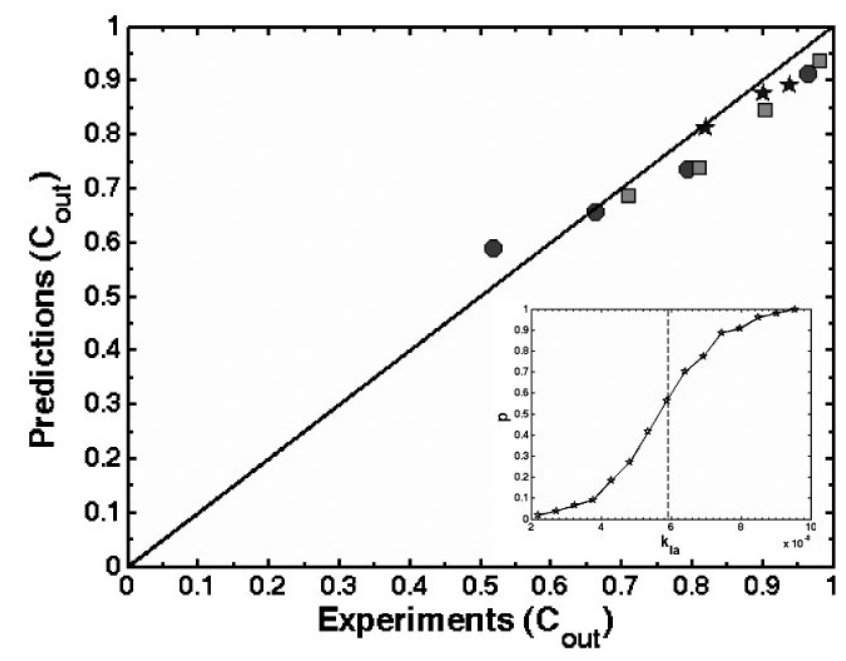

Figure 3. Determination of the mass transfer coefficient $k_{\text {la }}$ from oxygen concentration measurements and the model. This figure shows the comparison of model and experimental data for three levels of cell density as indicated earlier. The experimentally measured scaled concentration of oxygen at the outlet of the reactor $(x=22.5 \mathrm{~mm}$; scaled w.r.t inlet concentration) is plotted as a function of the predicted values for three cell densities circles-high $\left(1.67 \times 10^{5} \mathrm{cells} / \mathrm{cm}^{2}\right)$, squares - medium $\left(5.2 \times 10^{4} \mathrm{cells} / \mathrm{cm}^{2}\right)$, and stars-low $\left(9.9 \times 10^{3} \mathrm{cells} / \mathrm{cm}^{2}\right)$. The experimental and the predicted values for all velocities are plotted with the agreement line $(y=x)$. The best fit value for $k_{\text {la }}$ was $5.98 \times 10^{-8} \mathrm{~m} / \mathrm{s}$. Three sets of 50 Monte-Carlo simulations was performed to ascertain the variation of $k_{\mathrm{la}}$ for measurement uncertainty in the velocity assuming normally distributed errors with standard deviation equal to $30 \%$ of the mean. The standard deviation of the best fit $k_{\mathrm{la}}$ values was found to be $1.5 \times 10^{-8} \mathrm{~m} / \mathrm{s}$. Inset shows the cumulative probability distribution for the best fit $k_{\text {la }}$ values for all simulations. Note that the mean value of $k_{\mathrm{la}}$ is centered, that is, has a probability of $50 \%$. The mean regression coefficient for all the runs was $88 \%$. All the experimental data taken in Section B were used for the regression, with the inlet concentration as the concentration measured at $x=17.5 \mathrm{~mm}$.

oxygen by cells. Hence, we used the partial differential equation model and the experimental data and formulated the optimization problem to estimate the overall mass transfer coefficient $\left(k_{\mathrm{la}}\right)$ from the experimental measurements from Section B. Figure 3 shows the results of the optimization and the model fit to the experimental data. The good fit of the model predictions and experimental data shows that the single parameter $\left(k_{\mathrm{la}}\right)$ film model of diffusive mass transfer of oxygen from PDMS surface is sufficient to explain the diffusion process in the microdevice.

The mean value of the overall mass transfer coefficient was found to be $5.98 \times 10^{-8} \mathrm{~m} / \mathrm{s}$ with standard deviation of $1.59 \times 10^{-8}$. The standard deviation for the measurement was estimated using the Monte Carlo procedure outlined earlier. Assuming normally distributed errors in the measured variable (the velocity $\langle u\rangle$ ), the fitting procedure was repeated to estimate the standard deviation in $k_{\mathrm{la}}$ and a plot of the probability distribution of $k_{\mathrm{la}}$ from the results of these runs is shown in the inset (Fig. 3). We saw that the probability distribution for $k_{\mathrm{la}}$ is similar to the imposed distribution of $\langle u\rangle$, and the measurement uncertainty of $\langle u\rangle$ is directly reflected in the variation of inferred values of
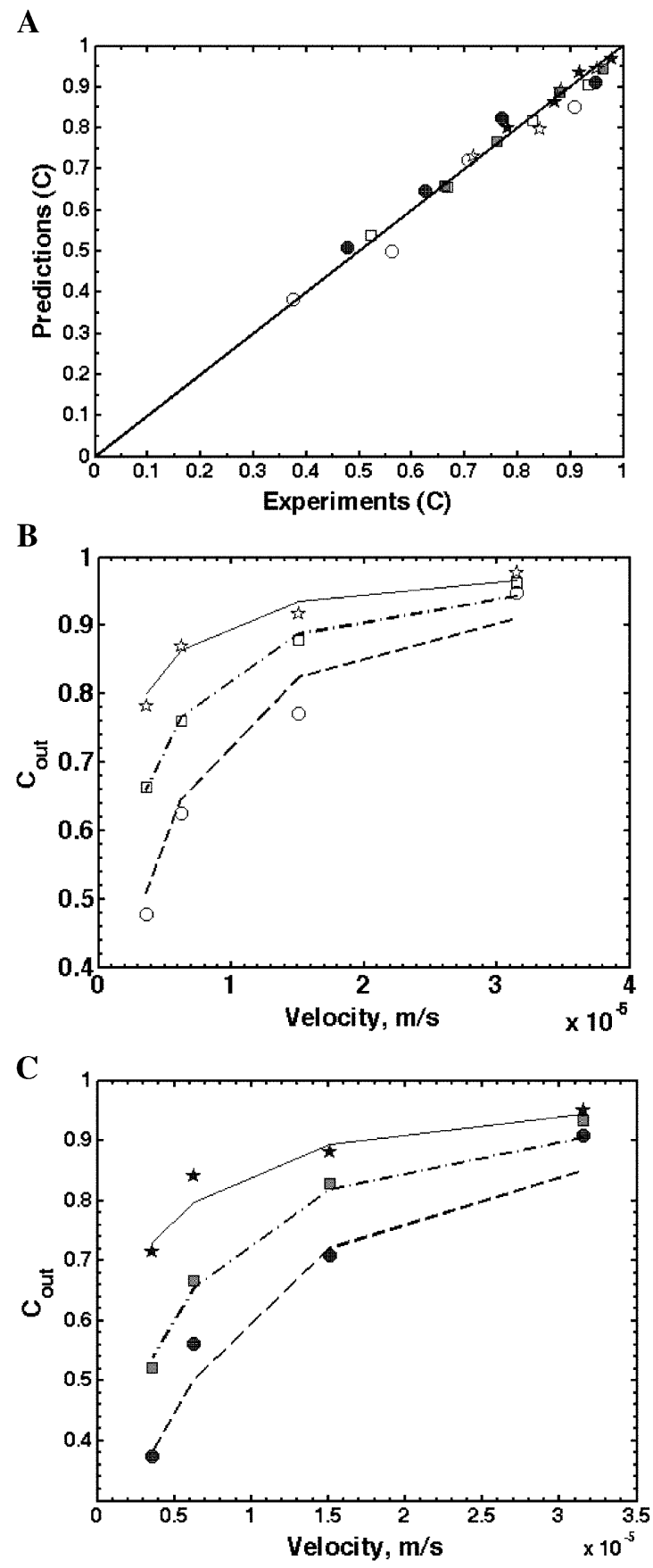

Figure 4. Comparison of predicted and observed oxygen concentrations for oxygen uptake model independent of cell density. A: Comparison of model and experimental data for three levels of cell density (circles, squares, and stars for high, medium, and low cell densities, respectively) and for both locations (filled markers for $x=10 \mathrm{~mm}$ and hollow markers for $x=17.5 \mathrm{~mm}$ ). The experimentally measured scaled concentration (scaled w.r.t inlet concentration) of oxygen at is plotted as a function of the predicted values. The experimental and the predicted values for all velocities are plotted with the agreement line $(y=x)$. B and C: Comparison of the model predicted (lines) and experimental (points) scaled oxygen concentrations in the device as a function of the media velocity at locations $x=10 \mathrm{~mm}$ and $x=17.5 \mathrm{~mm}$, respectively. The experimental data in Section A at three locations, $x=10 \mathrm{~mm}$ and $x=17.5 \mathrm{~mm}$ and the inlet, was used for the regression. The data for each cell density were fitted individually, hence a total of eight points were used to infer two parameters. Inferred values of $V_{\max }$ and $K_{\mathrm{m}}$ along with the statistics for regression can be found in Table I. 
$k_{\text {la }}$. Interestingly, this approach can be used to develop experimental protocols where the measurement accuracy for particular variable is designed to achieve desired accuracy in the inferred parameter.

The estimated value of $k_{\mathrm{la}}$ is lower than from what one would expect from virgin PDMS, and we attribute it to the fact that the PDMS used for our experiments is plasmatreated, which is known to lower the oxygen permeability (Houston et al., 2002; Shiku et al., 2006). The dataset consists of multiple measurements with different devices and cell cultures of different cell densities and hence the standard deviation is also indicative of the reproducibility of the device-making process.

\section{Quantification of Cellular Uptake Rates for Experimental Cell Densities}

We inferred the oxygen uptake rate parameters $\left(V_{\max }\right.$ and $K_{\mathrm{m}}$ ) for three different values of cell density individually as per the uptake model described by Equation (9a) using measured data (Fig. 2) from Section A of the device. The goodness of the fit, as indicated by the comparison of model and experimental results, is shown in Figure 4. The model is in good agreement of the experimental data (regression coefficients $>92 \%$ ).

Table I shows the inferred values of $K_{\mathrm{m}}$ and $V_{\max }$ for the three different cell densities. The standard deviations in $K_{\mathrm{m}}$ and $V_{\max }$ for each of the three cell densities are determined by the Monte Carlo procedure. We found that the values of $V_{\max }$ vary systematically with cell density. While the values of $K_{\mathrm{m}}$ also vary with the cell density, but the large standard deviations for this parameter suggest uncertainty in its values. However, for the current experimental data, the concentrations of oxygen are much larger than the $K_{\mathrm{m}}$ values $\left(K_{\mathrm{m}}<c\right)$, and hence the specific oxygen uptake rate is largely determined by $V_{\max }$ (Eq. 9a).

We found that the OUR for HepG2 cells varies between 1 and $9 \times 10^{-17} \mathrm{~mol} / \mathrm{cell} / \mathrm{s}$ depending on the cell density. This rate is similar to that found by other researchers for HepG2 cells via other measurement methods (Chin et al., 2008; Liu et al., 1991). Furthermore, we found that the dependence of $V_{\max }$ and hence the specific oxygen uptake of cells on the cell density is in qualitative agreement with observations of other researchers for other cell types (Cho et al., 2007; Rotem et al., 1992).

\section{Quantification of Cellular Uptake Rates for Unified Model of Oxygen Uptake}

The results in the previous section indicated that the specific oxygen uptake rate is a function of cell culture density. At higher cell densities, cells tend to grow more slowly and thus have lower metabolic activity; we found a lower specific oxygen consumption rate. A logistic term, which is generally used to model the dependence of the cell proliferation/ growth rate on the instantaneous cell density, hence may explain the dependence of our inferred cell uptake parameter on the cell culture density. We, therefore, repeated the inference procedure with the unified model described by Equation (9b).

The results for the fit with the new model are shown in Figure 5. The inferred values for the parameters are shown in Table I. Again, the value of $V_{\max }$, which dominates the specific oxygen uptake rate $\left(\right.$ as $\left.K_{\mathrm{m}}<c\right)$ is in accordance with the range of values reported previously in literature (Chin et al., 2008; Liu et al., 1991). The value of maximum cell density $\phi_{\max }$ is in agreement with our experimentally observed maximum cell density in the microchannel.

While there is an additional parameter $\left(\phi_{\max }\right)$ for the model, we now simultaneously fit the data for all three cell densities (Fig. 5). The regression coefficient for the new model is lower than the earlier model; however a direct comparison of the two models is not possible by just considering the regression coefficient, as they fit different numbers of datapoints, and also have different numbers of parameters. Hence to verify if the addition of a new parameter is justified, we used the Bayesian information criterion and found the BIC score for our model $(-55)$ is lower than the scores of the model for three individual cell densities $(-20,-27$, and -25$)$ justifying the addition of the new parameter. The results of this analysis indicate that indeed, the cellular uptake of oxygen is dependent on the total cell density, and hence it should be considered while designing the oxygen supply for the microfluidic device.

Table I. Inferred parameter values and regression statistics. ${ }^{a}$

\begin{tabular}{|c|c|c|c|c|c|c|}
\hline & $\begin{array}{l}\text { Cell density, } \phi \\
\left(\times 10^{3} \text { cells } / \mathrm{cm}^{2}\right)\end{array}$ & $\begin{array}{c}V_{\max } \\
\left(\times 10^{17} \mathrm{~mol} / \mathrm{cell} / \mathrm{s}\right)\end{array}$ & $\begin{array}{c}K_{m} \\
\left(\mathrm{~mol} / \mathrm{m}^{3}\right)\end{array}$ & $\begin{array}{l}R^{2} \\
(\%)\end{array}$ & BIC & $\begin{array}{c}\phi_{\max } \\
\left(\times 10^{3} \text { cells } / \mathrm{cm}^{2}\right)\end{array}$ \\
\hline \multirow{3}{*}{ Model I } & 167 & $\begin{array}{c}1.12 \\
{[0.32]}\end{array}$ & $\begin{array}{c}0.12 \\
{[0.10]}\end{array}$ & 95 & -20 & - \\
\hline & 52 & $\begin{array}{c}1.83 \\
{[0.57]}\end{array}$ & $\begin{array}{c}0.07 \\
{[0.09]}\end{array}$ & 98 & -27 & - \\
\hline & 9.9 & $\begin{array}{l}5.74 \\
{[1.6]}\end{array}$ & $\begin{array}{c}0.11 \\
{[0.11]}\end{array}$ & 94 & -25 & - \\
\hline Model II & ALL & $\begin{array}{l}6.62 \\
{[1.2]}\end{array}$ & $\begin{array}{c}0.154 \\
{[0.087]}\end{array}$ & 87 & -55 & $\begin{array}{c}254 \\
{[3.4]}\end{array}$ \\
\hline
\end{tabular}

\footnotetext{
${ }^{a}$ The values inside the brackets [ ] below the reported value indicate the standard deviation in the base value ascertained by Monte Carlo simulations with
} three sets of 50 simulations each. 


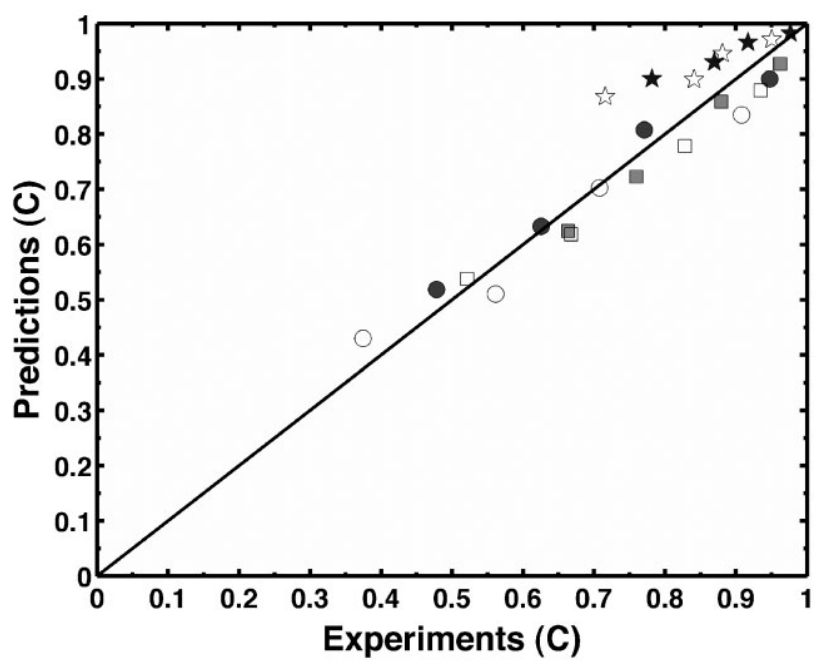

Figure 5. Model results with logistic growth factor densities for the proposed oxygen uptake model. Figure shows the comparison of model and experimental data for three levels of cell density; High (circles), Medium (squares), and Low (stars) and for both locations (filled markers for $x=10 \mathrm{~mm}$ and hollow markers for $x=17.5 \mathrm{~mm}$ ). The experimental data in Section A at three locations, $x=10 \mathrm{~mm}$ and $x=17.5 \mathrm{~mm}$ and the inlet, was used for the regression. The data for all cell density was fitted simultaneously, hence a total of 32 points were used to infer three parameters. Inferred values of $V_{\max }$ and $K_{\mathrm{m}}$ along with the statistics for regression can be found in Table I.

\section{Conclusion}

The motivation of this work was to develop a methodology to quantitatively measure key cellular parameters in a specified media environment. We developed a microfluidic device-based assay and accompanying model and inference procedure to determine the cellular uptake rate of oxygen. By avoiding the simultaneous inference of both the uptake and the diffusion parameters, the method used here minimizes errors arising due to the correlation of the parameters. The independent inference of the mass transfer coefficient is critical to the accurate inference of the cellular uptake parameters. We also demonstrated a dependence of oxygen uptake on cell density and characterized a new uptake model that accounts for this. Our model and associated parameter values can be used for the design or optimization of microfluidic cell culture reactors. The method and the device can be readily adapted to measure uptake rates of other soluble factors (e.g., nutrients, cell secreted signaling molecules) and also can be extended to other systems/geometries. Furthermore, the use of modelbased sensitivity studies as the Monte Carlo simulations described here can enable the identification of variables that need to be measured with specified accuracy to target a particular accuracy of an inferred parameter of interest. We believe that the combination of models with new experimental devices can help us develop novel, more accurate assays to measure cellular properties of interest.
This work was supported by US Army Research Laboratories and Research Office Grant DAAD 19-03-1-0168. We thank Jay Soong-Jin Lee, Ji Sun Sunny Choi, and Amy Oberlin for help with experiments and Peter Woolf for useful discussions.

\section{References}

Alper JS, Gelb RI. 1991. Monte Carlo method for the determination of confidence intervals: Analysis of nonnormally distributed errors in sequential experiments. J Phys Chem 95:104-108.

Andersson H, van den Berg A. 2004. Microfabrication and microfluidics for tissue engineering: State of the art and future opportunities. Lab Chip 4:98-103.

Balis UJ, Behnia K, Dwarakanath B, Bhatia SN, Sullivan SJ, Yarmush ML, Toner M. 1999. Oxygen consumption characteristics of porcine hepatocytes. Metab Eng 1:49-62.

Bates DM, Watts DG. 1988. Nonlinear regression analysis and its applications. Hoboken, NJ: John Wiley \& Sons.

Bird BR, Stewart WE, Lightfoot EN. 2001. Transport phenomena. New York: John Wiley \& Sons.

Burnham KP, Anderson DR. 2002. Model selection and multi-model inference-A practical information-theoretic approach. Berlin: Springer. 488 p.

Chin K, Khattak SF, Bhatia SR, Roberts SC. 2008. Hydrogel-perfluorocarbon composite scaffold promotes oxygen transport to immobilized cells. Biotechnol Prog 24:358-366.

Cho CH, Park J, Nagrath D, Tilles AW, Berthiaume F, Toner M, Yarmush ML. 2007. Oxygen uptake rates and liver-specific functions of hepatocyte and 3T3 fibroblast co-cultures. Biotechnol Bioeng 97:188-199.

Donaldson JR, Schnabel RB. 1987. Computational experience with confidence regions and confidence intervals for nonlinear least squares. Technometrics 29:67-82.

Fisher RJ, Peattie RA. 2007. Controlling tissue microenvironments: Biomimetics, transport phenomena, and reacting systems. Adv Biochem Eng Biotechnol 103:1-73.

Foy BD, Rotem A, Toner M, Tompkins RG, Yarmush ML. 1994. A device to measure the oxygen uptake rate of attached cells: Importance in bioartificial organ design. Cell Transplant 3:515-527.

Futai N, Gu W, Song JW, Takayama S. 2006. Handheld recirculation system and customized media for microfluidic cell culture. Lab Chip 6:149154.

Ghanem A, Shuler ML. 2000. Characterization of a perfusion reactor utilizing mammalian cells on microcarrier beads. Biotechnol Prog 16:471-479.

Gu W, Zhu X, Futai N, Cho BS, Takayama S. 2004. Computerized microfluidic cell culture using elastomeric channels and Braille displays. Proc Natl Acad Sci USA 101:15861-15866.

Guarino RD, Dike LE, Haq TA, Rowley JA, Pitner JB, Timmins MR. 2004. Method for determining oxygen consumption rates of static cultures from microplate measurements of pericellular dissolved oxygen concentration. Biotechnol Bioeng 86:775-787.

Houston KS, Weinkauf DH, Stewart FF. 2002. Gas transport characteristics of plasma treated poly(dimethylsiloxane) and polyphosphazene membrane materials. J Membr Sci 205:103-112.

Hynes J, Hill R, Papkovsky DB. 2006. The use of a fluorescence-based oxygen uptake assay in the analysis of cytotoxicity. Toxicol In Vitro 20:785-792.

Jorjani P, Ozturk SS. 1999. Effects of cell density and temperature on oxygen consumption rate for different mammalian cell lines. Biotechnol Bioeng 64:349-356.

Kang L, Chung BG, Langer R, Khademhosseini A. 2008. Microfluidics for drug discovery and development: From target selection to product lifecycle management. Drug Discov Today 13:1-13.

Liu JJ, Chen BS, Tsai TF, Wu YJ, Pang VF, Hsieh A, Hsieh JH, Chang TH. 1991. Long term and large-scale cultivation of human hepatoma Hep G2 cells in hollow fiber bioreactor. Cultivation of human hepatoma Hep G2 in hollow fiber bioreactor. Cytotechnology 5:129-139. 
Mehta K, Linderman JJ. 2006. Model-based analysis and design of a microchannel reactor for tissue engineering. Biotechnol Bioeng 94: 596-609.

Mehta G, Mehta K, Sud D, Song JW, Bersano-Begey T, Futai N, Heo YS, Mycek MA, Linderman JJ, Takayama S. 2007. Quantitative measurement and control of oxygen levels in microfluidic poly(dimethylsiloxane) bioreactors during cell culture. Biomed Microdevices 9:123-134.

Mehta G, Torisawa T, Takayama S. 2008. Engineering cellular microenviroments with microfluidics. In: Gomez F, editor. Biological applications of microfluidics. Hoboken, NJ: Wiley-InterScience. p. 87-114.

Nahmias Y, Berthiaume F, Yarmush ML. 2007. Integration of technologies for hepatic tissue engineering. Adv Biochem Eng Biotechnol 103:309_ 329.

Ostrovidov S, Jiang J, Sakai Y, Fujii T. 2004. Membrane-based PDMS microbioreactor for perfused $3 \mathrm{D}$ primary rat hepatocyte cultures. Biomed Microdevices 6:279-287.

Park J, Berthiaume F, Toner M, Yarmush ML, Tilles AW. 2005. Microfabricated grooved substrates as platforms for bioartificial liver reactors. Biotechnol Bioeng 90:632-644.

Puleo CM, Yeh HC, Wang TH. 2007. Applications of MEMS technologies in tissue engineering. Tissue Eng 13:2839-2854.

Rotem A, Toner M, Tompkins RG, Yarmush ML. 1992. Oxygen uptake rates in cultured rat hepatocytes. Biotechnol Bioeng 40:1286-1291.
Roy P, Baskaran H, Tilles AW, Yarmush ML, Toner M. 2001. Analysis of oxygen transport to hepatocytes in a flat-plate microchannel bioreactor. Ann Biomed Eng 29:947-955.

Sadegh Zadeh K, Montas HJ, Shirmohammadi A. 2006. Identification of biomolecule mass transport and binding rate parameters in living cells by inverse modeling. Theor Biol Med Model $3: 36$.

Shiku H, Saito T, Wu C, Yasukawa T, Yokoo M, Abe H, Matsue T, Yamada H. 2006. Oxygen permeability of surface-modified poly(dimethylsiloxane) characterized by scanning electrochemical microscopy. Chem Lett 35:234-235.

Sin A, Chin KC, Jamil MF, Kostov Y, Rao G, Shuler ML. 2004. The design and fabrication of three-chamber microscale cell culture analog devices with integrated dissolved oxygen sensors. Biotechnol Prog 20:338345 .

Situma C, Hashimoto M, Soper SA. 2006. Merging microfluidics with microarray-based bioassays. Biomol Eng 23:213-231.

Sud D, Mehta G, Mehta K, Linderman J, Takayama S, Mycek MA. 2006. Optical imaging in microfluidic bioreactors enables oxygen monitoring for continuous cell culture. J Biomed Opt 11:050504.

Viravaidya K, Sin A, Shuler ML. 2004. Development of a microscale cell culture analog to probe naphthalene toxicity. Biotechnol Prog 20:316323. 